\title{
The expert COVID-19 team for older persons of the Quebec Health and Social Services Ministry
}

\author{
Olivier Beauchet $^{1,2,3,4}$
}

Received: 20 May 2020 / Accepted: 3 June 2020 / Published online: 16 June 2020

C) Springer Nature Switzerland AG 2020

\section{To the Editor:}

Recently, Hogan et al. underscored the key role played by geriatricians in the COVID-19 pandemic in Canada [1]. They asked the question about "... what impact the Coronavirus disease 2019 (COVID-19) pandemic will have on the future practice of geriatrics in Canada". In Quebec, which is the Canadian province that is most affected by the COVID19 pandemic, geriatricians and gerontologists are working in partnership with the Health and Social Services Ministry, as well as with the Older Persons and Caregivers Ministry to find solutions for better care of Quebec's older population in the short, medium and long terms [2]. This partnership was identified by the Quebec government early in the COVID19 pandemic as an action of central importance due to the numerous Quebec long-term care (LTC) facilities struggling under the weight of the COVID-19 pandemic, with at least 150 of them reporting confirmed COVID-19 cases in LTC on April 6, 2020 [3]. Older persons, especially frail older adults living in long-term care facilities are significantly at increased risk of contracting COVID-19 and developing severe complications [1-3]. They are also at risk for morbidity decompensation due to the breakdown of their care continuum caused by COVID-19 health care reorganization

Olivier Beauchet

olivier.beauchet@mcgill.ca

1 Department of Medicine, Division of Geriatric Medicine, Sir Mortimer B. Davis-Jewish General Hospital and Lady Davis Institute for Medical Research, McGill University, 3755 chemin de la Côte-Sainte-Catherine, Montréal, QC H3T 1E2, Canada

2 Dr. Joseph Kaufmann Chair in Geriatric Medicine, Faculty of Medicine, McGill University, Montreal, QC, Canada

3 Centre of Excellence on Longevity of McGill integrated University Health and Social Services Network, Montreal, QC, Canada

4 Lee Kong Chian School of Medicine, Nanyang Technological University, Singapore, Singapore and the homebound confinement. Social distancing, which includes physical distancing and being homebound, is used to describe the practice of maintaining space between individuals and is an effective intervention to reduce the spread of COVID-19 [4]. Nevertheless, this key prevention strategy may provoke adverse consequences in the older communitydwelling population [5].

Quebec is a vast Canadian province where the population is aging rapidly compared to other provinces. Quebec has the highest number of confirmed COVID-19 cases in Canada. As of May 18, 2020, there were 43,627 COVID-19 cases in Quebec. To ensure the control of the COVID-19 spread in older persons, the Minister of Health and Social Services, Danielle McCann, and the Minister for Older Persons and Caregivers, Marguerite Blais, have appointed a geriatrician as special advisor on April 10, 2020 [3]. This geriatrician works with a team of COVID-19 experts composed of geriatricians, gerontologists, public health and nursing specialists. This team also has the support and advices of two civil servants of the Ministry's staff. The Expert COVID-19 Team (ECT) provides advices (i.e., delay of answer $\leq 24 \mathrm{~h}$ ) and recommendations (i.e., delay of answer $\geq 24 \mathrm{~h}$ ) on the best preventive and curative interventions for older persons. Advices are answers for urgent questions asked by the Health and Social Services Ministry. Whereas, recommendations are (1) answers for non-urgent questions asked by the Health and Social Services Ministry or (2) ECT's suggestions for curative or preventive actions related to COVID-19 issues and concerns reported by experts of the ECT. In the case of recommendations, the ECT may receive inputs from a fieldbased advisory group composed of clinicians, researchers and members of community associations providing services and care for older community dwellers. Advices and recommendations are submitted directly to the Minister of Health and Social Services, and the Minister of the Older Persons and Caregivers. They are in turn analyzed at the Ministry level by a special coordination committee which is at the interface of all directorships and branches of the Ministry. 
This committee confirms relevance, non-duplication and complementary actions with Ministry's COVID-19 decisions. It can also propose changes if needed.

The special advisor and the ECT are one of Quebec solutions to strongly advocate for older persons during the COVID-19 pandemic. Both the special advisor and the ECT work towards being active in promoting advance care planning and are involved in the development of Quebec health policies for older persons. Noteworthy is the fact that all three of these points have been identified as key by Hogan et al. [1].

Acknowledgements The author acknowledges the members of the Expert COVID Team for their help in writing the manuscript.

Author contributions OB wrote the manuscript.

\section{Compliance with ethical standards}

Conflict of interest The author declares no conflict of interest.

Ethical approval This study is not an original study and does not involve human participants and animals.

Informed consent For this type of study, consent is not required.

\section{References}

1. Hogan DB, MacKnight C, Madden KM et al (2020) Canadian geriatrics in the time of COVID-19. J Am Geriatr Soc. https://doi. org/10.1111/jgs. 16518

2. Coronavirus Disease 2019 (COVID-19). Daily Epidemiology Update (Updated May 19, 2020, 8:00 AM ET). https://www. canada.ca/en/public-health/services/diseases/2019-novel-coron avirus-infection.html?utm_campaign $=\mathrm{gc}$-hc-sc-coronavirus 2021 -ao-2021-0005-9834796012\&utm_medium = search\&utm_sourc e = google_grant-ads-107802327544\&utm_content $=$ texten-434601690158\&utm_term $=\% 2$ Bcoronavirus\#a 1

3. Pandémie de la COVID-19-Mesures additionnelles pour protéger les personnes aînées (2:00 ET) http://www.fil-information.gouv. qc.ca/Pages/Article.aspx?idArticle $=2804109809$. Accessed 15 May 2020

4. Nickel CH, Rueegg M, Pargger H et al (2020) Age, comorbidity, frailty status: effects on disposition and resource allocation during the COVID-19 pandemic. Swiss Med Wkly 150:w20269

5. Hubbard RE, Maier AB, Hilmer SN et al (2020) Frailty in the Face of COVID-19. Age Ageing 6:afaa095. https://doi.org/10.1093/ ageing/afaa095

Publisher's Note Springer Nature remains neutral with regard to jurisdictional claims in published maps and institutional affiliations. 